\title{
Scaling properties of adsorption energies for hydrogen-containing molecules on transition-metal surfaces
}

Abild-Pedersen, Frank; Greeley, Jeffrey Philip; Studt, Felix; RossmeisI, Jan; Fronczek-Munter, Ture Rønved; Moses, Poul Georg; Skulason, Egill; Bligaard, Thomas; Nørskov, Jens Kehlet

Published in:

Physical Review Letters

Link to article, DOI:

10.1103/PhysRevLett.99.016105

Publication date:

2007

Document Version

Publisher's PDF, also known as Version of record

Link back to DTU Orbit

Citation $(A P A)$ :

Abild-Pedersen, F., Greeley, J. P., Studt, F., Rossmeisl, J., Fronczek-Munter, T. R., Moses, P. G., Skulason, E., Bligaard, T., \& Nørskov, J. K. (2007). Scaling properties of adsorption energies for hydrogen-containing molecules on transition-metal surfaces. Physical Review Letters, 99(1), 016105.

https://doi.org/10.1103/PhysRevLett.99.016105

\section{General rights}

Copyright and moral rights for the publications made accessible in the public portal are retained by the authors and/or other copyright owners and it is a condition of accessing publications that users recognise and abide by the legal requirements associated with these rights.

- Users may download and print one copy of any publication from the public portal for the purpose of private study or research.

- You may not further distribute the material or use it for any profit-making activity or commercial gain

- You may freely distribute the URL identifying the publication in the public portal 


\title{
Scaling Properties of Adsorption Energies for Hydrogen-Containing Molecules on Transition-Metal Surfaces
}

\author{
F. Abild-Pedersen, J. Greeley, F. Studt, J. Rossmeisl, T. R. Munter, P. G. Moses, E. Skúlason, T. Bligaard, and J. K. Nørskov \\ Center for Atomic-scale Materials Design, Department of Physics, NanoDTU, Technical University of Denmark, \\ DK-2800 Lyngby, Denmark \\ (Received 13 February 2007; published 6 July 2007)
}

\begin{abstract}
Density functional theory calculations are presented for $\mathrm{CH}_{x}, x=0,1,2,3, \mathrm{NH}_{x}, x=0,1,2, \mathrm{OH}_{x}, x=$ 0,1 , and $\mathrm{SH}_{x}, x=0,1$ adsorption on a range of close-packed and stepped transition-metal surfaces. We find that the adsorption energy of any of the molecules considered scales approximately with the adsorption energy of the central, $\mathrm{C}, \mathrm{N}, \mathrm{O}$, or $\mathrm{S}$ atom, the scaling constant depending only on $x$. A model is proposed to understand this behavior. The scaling model is developed into a general framework for estimating the reaction energies for hydrogenation and dehydrogenation reactions.
\end{abstract}

PACS numbers: 82.45.Jn

The formation of a bond between a molecule and a metal surface is an important phenomenon in a number of processes including heterogeneous catalysis [1], contact formation in molecular electronics [2], and anchoring of biomolecules to solids for sensors and other biomedical applications [3]. The adsorption energy is a key quantity describing the strength of the interaction of molecules with the surface. The adsorption energy can be measured by advanced surface science techniques [4-6]. Alternatively, density functional theory (DFT) offers the possibility of calculating adsorption energies with reasonable accuracy [7-11]. While both experiments and DFT calculations are feasible for a limited number of systems, they can hardly be performed in detail for all potentially interesting adsorption systems. There is therefore a need for simple models with the ability to estimate bond energies in a first screening of interesting systems. A successful model will also expose the important factors determining the strength of an adsorbate-surface bond. In the present Letter we will develop such a model for hydrogen-containing molecules. We use DFT calculations to derive a number of correlations between adsorption energies, and we then present a model to explain them. The model shows how the adsorbate valency, together with the properties of the $d$ electrons of the surface, determines the adsorption energy. We further develop the scaling model into a method for estimating hydrogenation or dehydrogenation reaction energies for organic molecules on transition-metal surfaces. The model is tested against full DFT calculations for reactions of hydrocarbons, alcohols, thiols, and amino acids.

First, we present results of extensive DFT calculations of the adsorption energies of $\mathrm{CH}_{x}, x=0,1,2,3, \mathrm{NH}_{x}, x=$ $0,1,2, \mathrm{OH}_{x}, x=0,1$, and $\mathrm{SH}_{x}, x=0,1$ on a range of close-packed and stepped metal surfaces. The study involves the close-packed fcc(111), fcc(100), hcp(0001), and bcc(110) surfaces, and the stepped fcc(211) and bcc(210) surfaces. Each of the surfaces is modeled by a $(2 \times 2)$ or a $(1 \times 2)$ surface unit cell for the close-packed and stepped surfaces, respectively. Each slab has a thickness of three layers in the direction perpendicular to the close-packed surface. These slabs are thick enough to capture the trends in the chemisorption energetics. The adsorbates and the topmost layer are allowed to relax fully in all configurations, and in the case of $\mathrm{Fe}, \mathrm{Ni}$, and $\mathrm{Co}$, spin polarization is taken into account. The binding energies of the different species have been taken for the most stable adsorption sites on all surfaces. The RPBE functional [12] in the generalized gradient approximation is used to describe exchange and correlation effects. The calculational method and setup is described in Ref. [13].

Figure 1 summarizes the results of the DFT calculations. We find for all the molecules studied that the adsorption energy of molecule $A \mathrm{H}_{x}$ is linearly correlated with the adsorption energy of atom $A$ :

$$
\Delta E^{A \mathrm{H}_{x}}=\gamma \Delta E^{A}+\xi .
$$

There is some scatter around the linear relations, but we note that while the adsorption energies vary by several electron volts over the range of metals considered here, the mean absolute error (MAE) is only $0.13 \mathrm{eV}$. Some of this scatter is related to differences in adsorption sites for adsorbates with different amounts of hydrogen. $\mathrm{CH}_{3}$, for instance, typically prefers a onefold adsorption site on the close-packed surfaces while $\mathrm{C}$ prefers the threefold site. If we were to use the adsorption energy of $\mathrm{C}$ in the onefold adsorption site as the reference, the quality of the correlation becomes significantly better $(\mathrm{MAE}=0.06 \mathrm{eV})$; see Fig. 2. We also find that when we use a reference with the same configuration as the molecule of interest, the scaling behavior includes alloys with the same accuracy as for the elemental metals (Fig. 2).

The main observation from Figs. 1 and 2 is that the slope of the linear relationship $\gamma$ in Eq. (1) is given to a good approximation by the number of $\mathrm{H}$ atoms in $A \mathrm{H}_{x}$ as $\gamma(x)=$ $\left(x_{\max }-x\right) / x_{\max }$, where $x_{\max }$ is the maximum number of $\mathrm{H}$ atoms that can bond to the central atom $A$ ( $x_{\max }=4$ for 

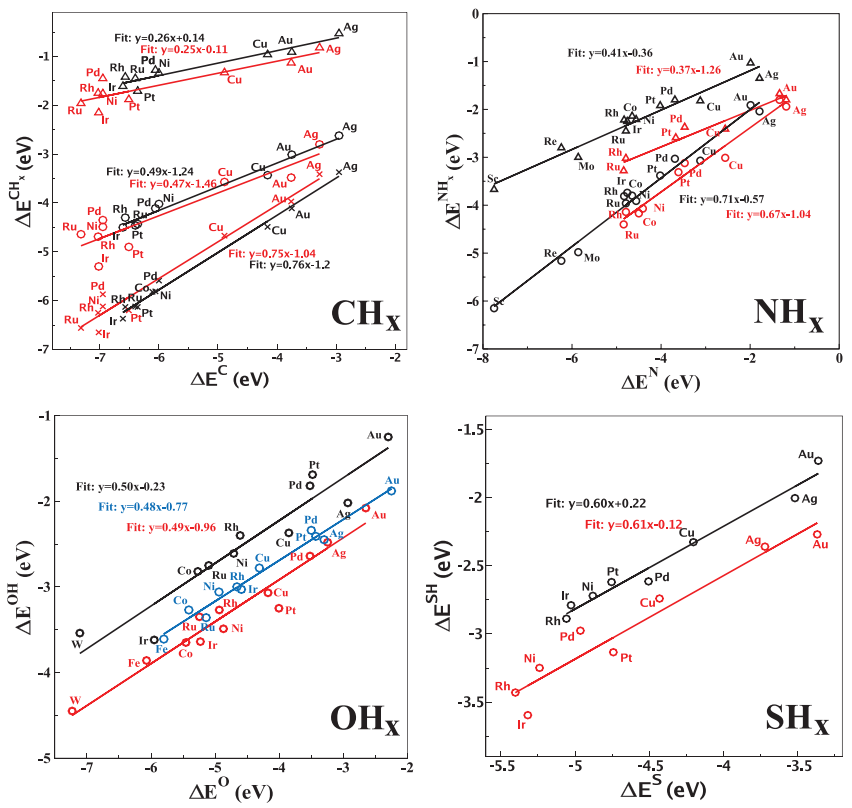

FIG. 1 (color). Adsorption energies of $\mathrm{CH}_{x}$ intermediates (crosses: $x=1$; circles: $x=2$; triangles: $x=3$ ), $\mathrm{NH}_{x}$ intermediates (circles: $x=1$; triangles: $x=2$ ), $\mathrm{OH}$, and $\mathrm{SH}$ intermediates plotted against adsorption energies of $\mathrm{C}, \mathrm{N}, \mathrm{O}$, and $\mathrm{S}$, respectively. The adsorption energy of molecule $A$ is defined as the total energy of $A$ adsorbed in the lowest energy position outside the surface minus the sum of the total energies of $A$ in vacuum and the clean surface. The data points represent results for close-packed (black) and stepped (red) surfaces on various transition-metal surfaces. In addition, data points for metals in the fcc(100) structure (blue) have been included for $\mathrm{OH}_{x}$.

$A=\mathrm{C}, x_{\max }=3$ for $A=N$, and $x_{\max }=2$ for $\left.A=\mathrm{O}, \mathrm{S}\right)$. Since $\left(x_{\max }-x\right)$ is the valency of the $A \mathrm{H}_{x}$ molecule, we conclude that for the four families of molecules considered the slope only depends on the valency of the adsorbate. In the following we will consider a model that allows us to understand the origin of this effect.

For some of the considered systems, simple valency or bond-counting arguments [14] can explain the results: Comparing $\mathrm{CH}, \mathrm{CH}_{2}$, and $\mathrm{CH}_{3}$ on the close-packed surfaces, we generally find $\mathrm{CH}$ (with a valency of 3 ) to prefer threefold adsorption sites, $\mathrm{CH}_{2}$ (valency of 2) to prefer twofold adsorption, and $\mathrm{CH}_{3}$ (valency of 1) to prefer onefold adsorption. The implication of these trends is that unsaturated bonds on the carbon atom form bonds to surface metal atoms; in effect, each unsaturated $s p^{3}$ hybrid on the central $\mathrm{C}$ atom binds independently to the $d$ states of the nearest neighbor metal atoms, consistent with the slopes in Fig. 1. However, this picture cannot include adsorbed atomic $\mathrm{C}$. Adsorbed $\mathrm{C}$ also adsorbs in a threefold site (neglecting long range reconstructions), but it does not have four bonds as would be needed to explain all the $\mathrm{C}$ data in Fig. 1. We also note that the overall scaling behavior is independent of the adsorption geometry and hence the details of the bonding; see Fig. 2. The scaling in Figs. 1 and 2 must therefore have a more general explanation that

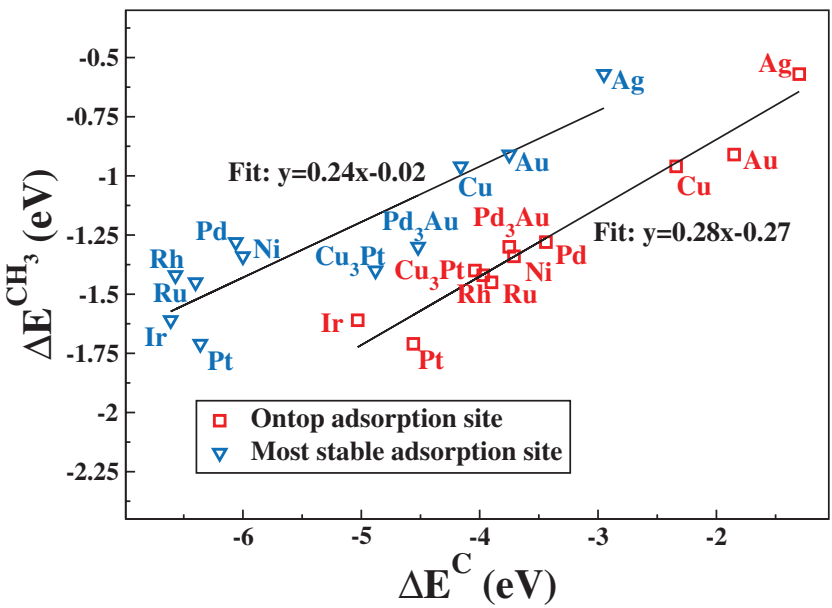

FIG. 2 (color). Binding energies of $\mathrm{CH}_{3}$ plotted against the binding energies of $\mathrm{C}$ for adsorption in the most stable sites (triangles) and in the case where both $\mathrm{CH}_{3}$ and $\mathrm{C}$ have been fixed in the on-top site (squares).

includes the argument above for $\mathrm{CH}, \mathrm{CH}_{2}$, and $\mathrm{CH}_{3}$ as a special case.

We will base our analysis on the $d$-band model which has been used quite successfully to understand trends in adsorption energies from one transition metal to the next [8,15-19]. According to the $d$-band model, it is useful to think of the formation of the adsorbate-surface bond as taking place in two steps. First, we let the adsorbate states interact with the transition metal $s p$ states, and then we include the extra contribution from the coupling to the $d$ states:

$$
\Delta E=\Delta E_{s p}+\Delta E_{d}
$$

The coupling to the metal $s p$ states usually contributes the largest part of the bonding and involves considerable hybridization and charge transfer. In terms of variations from one transition metal to the next it can, however, be considered to be essentially a constant; the $s p$ bands are broad, and all the transition metals have one $s p$ electron per metal atom in the metallic state [20]. According to the $d$-band model, the main contribution to the variations in bond energy from one transition metal to the next comes from the coupling to the metal $d$ states; the $d$ states form narrow bands of states close to the Fermi level, and the width and energy of the $d$ bands vary substantially between transition metals. According to the $d$-band model, all the variations among the metals observed in Fig. 1 should therefore be given by $\Delta E_{d}$. That means that the $x$ dependence of $\Delta E^{A \mathrm{H}_{x}}(x)$ must be given by the $d$ coupling alone: Let us assume for the moment that the $d$ coupling for $A \mathrm{H}_{x}$ is proportional to the valency parameter $\gamma$ defined above:

$$
\Delta E_{d}^{A \mathrm{H}_{x}}=\gamma(x) \Delta E_{d}^{A}
$$

Using Eq. (1), this will lead to the kind of relationship in Fig. 1. We can write the adsorption energy of molecule $A \mathrm{H}_{x}$ in terms of the adsorption energy of molecule $A$ as 


$$
\begin{aligned}
\Delta E^{A \mathrm{H}_{x}} & =\Delta E_{d}^{A \mathrm{H}_{x}}+\Delta E_{s p}^{A \mathrm{H}_{x}}=\gamma(x) \Delta E_{d}^{A}+\Delta E_{s p}^{A \mathrm{H}_{x}} \\
& =\gamma(x) \Delta E^{A}+\xi
\end{aligned}
$$

where $\xi=\Delta E_{s p}^{A \mathrm{H}_{x}}-\gamma(x) \Delta E_{s p}^{A}$ is independent of the metal in question. The parameter $\gamma$ can be read off Fig. 1 for each $A \mathrm{H}_{x} / A$ combination; see Eq. (1). The parameter $\xi$ can be obtained from calculations on any transition metal. In the following all model data presented are obtained using $\mathrm{Pt}(111)$ as the reference system.

The basic question remains, why the coupling to the $d$ states should scale with the valency of the adsorbate as in Eq. (1). We cannot provide a general rigorous proof of the scaling, Eq. (1), and most likely no such proof exists - the scatter in Fig. 1 indicates that the linear relationship is only approximate. What we will do, however, is show that Eq. (1) should hold approximately for the kind of systems investigated in Fig. 1.

The coupling of the adsorbate states to the $d$ band has two contributions [15,16,21], $\Delta E=\Delta E_{d}^{\text {hyb }}+\Delta E_{d}^{\text {orth. The }}$ first term describes the energy change associated with the formation of bonding and antibonding states. Since the $d$ coupling can often be described in second order perturbation theory [8,15,21], we can write $\Delta E_{d}^{\text {hyb }} \propto \Delta E_{d}^{\text {orth }} \propto V_{a d}^{2}$, where $V_{a d}$ is the Hamiltonian matrix element between the adsorbate and the metal $d$ states [21]. If there is more than one adsorbate state, the total interaction energy will scale with the sum of contributions from the adsorbate states, $V_{a d}^{2}=\sum_{i} V_{a_{i} d}^{2}$. We suggest that $V_{a d}^{2} \propto \gamma$ for the kind of systems considered in Fig. 1, and this directly gives the relation of Eq. (1). The reason is given in the following.

The coupling strength $V_{a d}^{2}\left(\left\{r_{a-i}\right\}\right)$ is a function of the number of metal neighbors and their distances to the adsorbate. Since the $d$ coupling is usually a minor perturbation to the bond energy, it is the $s p$ coupling which primarily determines the adsorption bond lengths, $r_{a-i}$. As $\mathrm{H}$ atoms are added to the central $\mathrm{C}, \mathrm{N}, \mathrm{O}$, or $\mathrm{S}$ atom, the adsorption bond lengths increase and the coupling strength decreases. The effective medium theory (EMT) [22] provides a simple way of quantifying this effect. In the EMT the bonding of an atom $A$ to other atoms in the vicinity is approximated by the interaction of $A$ with a homogeneous electron gas (the effective medium) of a density given by a spherical average $n$ of the density provided by the surrounding atoms: $\Delta E=\Delta E_{\mathrm{hom}}(n)$.

Such a local density approximation for the interaction energy gives a good description of general trends in bonding, including bond lengths of atoms in metals, adsorbates on metal surfaces, and of molecules [22-24]. The energy of embedding an atom in a homogeneous electron gas, $\Delta E_{\text {hom }}(n)$, generally has a minimum for a particular electron density, $n_{0}$, and the equilibrium geometry of atom $A$ is given by the position where $A$ experiences the optimum electron density, $n=n_{0}$.

Consider for instance a $\mathrm{C}$ atom outside a metal surface. The adsorption bond length is given by the distance outside the surface where the electron density from the surface around the $\mathrm{C}$ atom is $n_{\text {surf }}=n_{0}$. Now add $\mathrm{H}$ atoms to the $\mathrm{C}$ atom. Each $\mathrm{H}$ atom will provide electron density to the $\mathrm{C}$ atom, and the electron density needed from the surface to reach $n=n_{0}$ is smaller. For a fixed adsorption site (one-, two-, or threefold), the bond length between the surface atoms and the $\mathrm{C}$ atom must therefore increase. Alternatively, the adsorption site can change as in the case of $\mathrm{CH}$, $\mathrm{CH}_{2}$, and $\mathrm{CH}_{3}$ discussed above. In that case we would then expect the $\mathrm{C}$-metal bond length to be independent of $x$, since the change in metal coordination number corresponds exactly to the increase in the $\mathrm{H}$ coordination number for this sequence of systems. This is precisely what we find in the calculations. Returning to the general case, the electron density from the surface $n_{\text {surf }}$ needed to obtain $n=n_{0}$ will continue to decrease as the number $x$ of $\mathrm{H}$ atoms increases. When $x=4$ the $\mathrm{H}$ atoms must contribute all the electron density needed for the central $\mathrm{C}$ atom, $4 n_{\mathrm{H}}=n_{0}$, since a methane molecule does not bind to the surface at all (neglecting van der Waals interactions). The density contribution from the surface at the equilibrium site for $\mathrm{CH}_{x}$ is therefore

$$
n_{\text {surf }}=\frac{\left(x_{\max }-x\right)}{x_{\max }} n_{0}=\gamma(x) n_{0} .
$$

The linear dependence on $x$ in Eq. (5) is based on the reasonable assumption that the contribution to the electron density is the same for all $x \mathrm{H}$ atoms, and that the total density should add up to $n_{0}$ [25]. The electron density $n$ can be viewed as a generalized bond order [26,27], and the requirement that $n=n_{0}$ is then an example of bond order conservation.

Since we are using EMT to model the $s p$ contribution to the bonding, $n_{\text {surf }}$ denotes the $s p$ electron density outside the surface. The decay length of $n_{\text {surf }}$ outside the surface is given asymptotically by the work function (the energy of the Fermi level relative to vacuum). Since the $d$ states have energies close to the Fermi level as well, their decay length is roughly the same. That means that to a first approximation $V_{a d}^{2}$ scales with $n_{\text {surf }}$. We have therefore shown that the following relations hold approximately,

$$
\Delta E_{d}(x) \propto V_{a d}^{2}(x) \propto n_{\text {surf }}(x) \propto \frac{x_{\max }-x}{x_{\max }}=\gamma(x),
$$

which implies Eq. (1).

Given the understanding provided above, we can try to generalize the findings in Fig. 1. For any hydrogenation or dehydrogenation reaction of molecules bonding to a transition-metal surface via $\mathrm{C}, \mathrm{N}, \mathrm{O}$, or $\mathrm{S}$ atoms, we should be able to estimate the reaction energy for all transition metals given the reaction energy for just one metal. For each atom $A_{i} i=1, \ldots, N$ bonding to the surface, we determine the change $\Delta \gamma_{i}$ in the valence parameter during the reaction, and we can then estimate variation in the bond energy for the full system from the variations in the bond energies of the $A_{i}$ : 


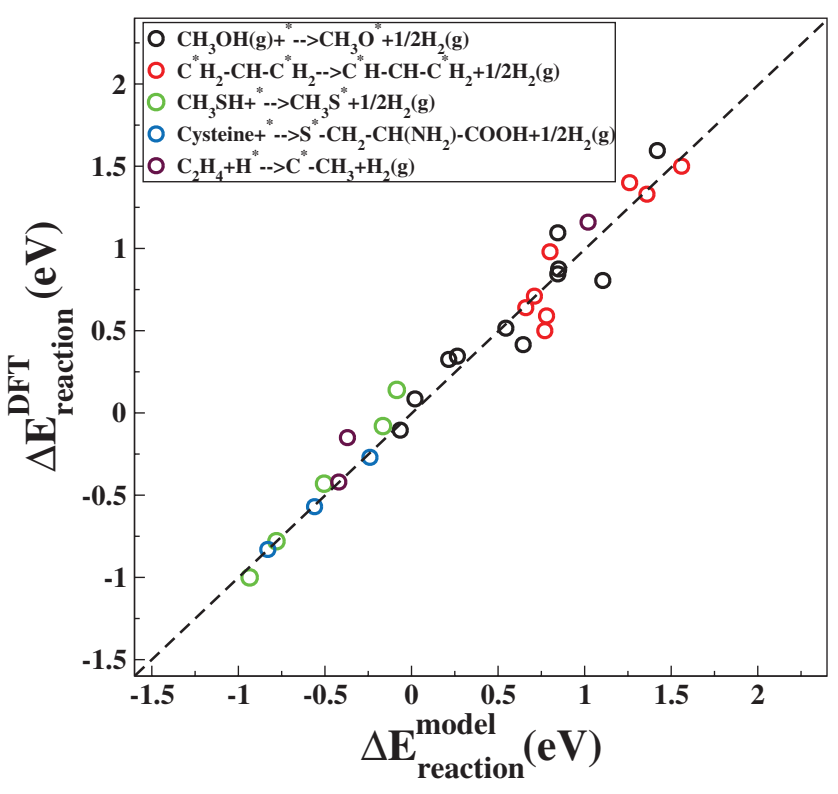

FIG. 3 (color). Calculated reaction energies for a series of dehydrogenation reactions plotted against the model predictions. The model data have been generated using calculated $\operatorname{Pt}(111)$ data as reference.

$$
\Delta E=\sum_{i=1}^{N}\left(\Delta \gamma_{i} \Delta E^{A_{i}}+\Delta \xi_{i}\right)=\sum_{i=1}^{N}\left(\Delta \gamma_{i} \Delta E^{A_{i}}\right)+\Delta \xi
$$

The change in the $\xi$ parameter for the particular reaction needs to be calculated once and for all by calculating the reaction energy for one single metal. In Fig. 3, we compare the model to complete DFT calculations for hydrogenation or dehydrogenation of a series of hydrocarbons, alcohols, thiols, and amino acids. In each case, we have calculated $\Delta \xi$ from $\mathrm{Pt}(111)$ data. The agreement between the model and the full DFT calculations indicates that the model has the power to describe both the absolute magnitude and the trends in reaction energies for hydrogenation or dehydrogenation reactions of a number of organic molecules on transition-metal surfaces. We note that the scaling relations can easily be generalized so that the adsorption energy of any hydrogenated species $A \mathrm{H}_{y}$ is used as the reference instead of $A$.

By combining the present model with the BrønstedEvans-Polanyi-type correlations that have been established between activation barriers and reaction energies for surface reactions $[8,10,11]$, it will be possible to estimate the full potential energy diagram for a surface catalyzed reaction for any transition metal on the basis of the $\mathrm{C}$, $\mathrm{N}, \mathrm{O}$, and $\mathrm{S}$ chemisorption energies and a calculation for a single metal. We suggest that this will be a useful tool in screening for new catalysts. Such estimates can subsequently be followed up by full DFT calculations and experiments for the most interesting systems.
The Center for Atomic-scale Materials Design is sponsored by the Lundbeck Foundation. Additional support from the Danish Research Councils and the Danish Center for Scientific Computing are also acknowledged.

[1] Z. Ma and F. Zaera, Surf. Sci. Rep. 61, 229 (2006).

[2] C. Joachim and M. Ratner, Proc. Natl. Acad. Sci. U.S.A. 102, 8801 (2005).

[3] B. Kasemo, Surf. Sci. 500, 656 (2002).

[4] G. Somorjai, Introduction to Surface Chemistry and Catalysis (Wiley, New York, 1994).

[5] W. A. Brown, R. Kose, and D. A. King, Chem. Rev. 98, 797 (1998).

[6] H. Gross, C. Campbell, and D. A. King, Surf. Sci. 572, 179 (2004).

[7] J. K. Nørskov, M. Scheffler, and H. Toulhoat, MRS Bull. 31, 669 (2006).

[8] B. Hammer and J. K. Nørskov, Adv. Catal. 45, 71 (2000).

[9] J. Greeley and M. Mavrikakis, J. Phys. Chem. B 109, 3460 (2005).

[10] V. Pallassana and M. Neurock, J. Catal. 191, 301 (2000).

[11] A. Michaelides et al., J. Am. Chem. Soc. 125, 3704 (2003).

[12] B. Hammer, L. Hansen, and J. K. Nørskov, Phys. Rev. B 59, 7413 (1999).

[13] T. Bligaard et al., J. Catal. 224, 206 (2004).

[14] G. Papoian, J. K. Nørskov, and R. Hoffmann, J. Am. Chem. Soc. 122, 4129 (2000).

[15] B. Hammer and J. K. Nørskov, Surf. Sci. 343, 211 (1995).

[16] B. Hammer and J. K. Nørskov, Nature (London) 376, 238 (1995).

[17] A. Eichler, F. Mittendorfer, and J. Hafner, Phys. Rev. B 62, 4744 (2000).

[18] J. Greeley and M. Mavrikakis, Nat. Mater. 3, 810 (2004).

[19] A. Roudgar and A. Gross, Phys. Rev. B 67, 033409 (2003).

[20] O. K. Andersen, O. Jepsen, and D. Glötzel, Highlights of Condensed Matter Theory (North-Holland, New York, 1985).

[21] B. Hammer and J. K. Nørskov, Theory of Adsorption and Surface Reactions (Kluwer, Dordrecht, 1997).

[22] J. K. Nørskov and N.D. Lang, Phys. Rev. B 21, 2131 (1980).

[23] M. Stott and E. Zaremba, Phys. Rev. B 22, 1564 (1980).

[24] M. Puska, R. Nieminen, and M. Manninen, Phys. Rev. B 24, 3037 (1981).

[25] $\mathrm{NH}_{3}, \mathrm{H}_{2} \mathrm{O}$, and $\mathrm{H}_{2} \mathrm{~S}$ do bind weakly to the surface. This bonding by lone pairs cannot be described in the simplest EMT model. The bond lengths to the surface of the fully hydrogenated species are, however, significantly larger than for any of the less hydrogenated species, and the density contribution from the surface is small. This is therefore a small correction to the scaling picture developed here.

[26] E. Shustorovich, Surf. Sci. 176, L863 (1986).

[27] A. T. Bell and E. Shustorovich, J. Catal. 121, 1 (1990). 